\title{
Gene profiling in the dynamic regulation of the lifespan of the myelin sheath structure in the optic nerve of rats
}

\author{
FANG XIE ${ }^{1,2^{*}}$, HAN FU $^{1 *}, \mathrm{JIU}_{-C O N G ~ Z H A N G}{ }^{3 *}$, XUE-FENG CHEN ${ }^{1}$, XIAO-LIANG WANG ${ }^{1}$ and JUN CHEN ${ }^{1}$ \\ ${ }^{1}$ Institute for Biomedical Sciences of Pain, Tangdu Hospital, The Fourth Military Medical University, Xi'an, Shaanxi 710038; \\ ${ }^{2}$ Institute of Basic Medical Sciences, Academy of Military Medical Science, Beijing 100039; ${ }^{3}$ Department of Gastroenterology, \\ Lanzhou General Hospital of Lanzhou Military Command, Lanzhou, Gansu 730050, P.R. China
}

Received July 24, 2013; Accepted April 1, 2014

DOI: $10.3892 / \mathrm{mmr} .2014 .2227$

\begin{abstract}
Aging of the nervous system leads to impairments in cognition and motor skills, and is a major risk factor for several neurological disorders. Recently, numerous nerve function deficits that appear with aging have been found to be a consequence of myelin abnormalities; however, the genetic mechanism of the age-related alterations in the myelin sheath has not yet been fully elucidated. In the present study, the morphology of the myelin sheath in the optic nerve of rats was analyzed at 10 time-points throughout life. Marked alterations in the myelin sheath were observed in aging and aged optic nerves, and these became progressively more severe with time. To determine the biological processes affected by aging in the myelin sheath, the age-related profiling of the myelin sheath in rat optic nerves was established using microarray hybridization at 10 time-points throughout life, between birth and senescence. From the results, 3,826 transcripts associated with the age-related alterations in the myelin sheath of the optic nerve were identified. It was found that the biological processes most significantly altered by aging were lipid metabolism, the immune response and transmitter transport. This suggests that the downregulation of lipid synthesis genes and the upregulation of immune and neurotransmitter transport genes in aging may be the genetic basis for the age-related alterations observed in the myelin sheath.
\end{abstract}

\section{Introduction}

Advances in biomedical sciences have substantially contributed to an increase in life expectancy over the past 50 years. However, this progress has led to the necessity for the treatment

Correspondence to: Dr Fang Xie, Institute for Biomedical Sciences of Pain, Tangdu Hospital, The Fourth Military Medical University, 1st Xinsi Road, Baqiao, Xi'an, Shaanxi 710038, P.R. China

E-mail:vancoxie@sina.com

*Contributed equally

Key words: aging, myelin sheath, gene profiling, neuropathy and prevention of emerging age-related disorders. As one of the major targets of senescence, the nervous system is vulnerable to changes in the environment that arise due to aging, and is involved in numerous age-dependent neurological deficits, including pain (1), impaired motor performance (2), cognitive decline (3) and dementia (4). In the past century, the majority of studies about brain aging have focused on the alterations in neurons and synapses in older individuals; however, little has been achieved in the prevention of age-related disorders. A number of neuroimaging studies have found that changes in white matter, particularly changes in the myelin sheath, may contribute to the age-dependent functional deficits observed in the nervous system $(5,6)$. Several species, including humans, non-human primates and rodents, have been observed to exhibit age-related myelin breakdown in the nervous system through ultrastructure electron microscopy studies $(7,8)$. Certain studies have found that changes in the nerve fibers and myelin sheath were affected by aging and were likely to have an important role in the development of age-related cognitive decline in humans and primates $(9,10)$. Furthermore, the development of age-related disorders, such as Alzheimer's and Parkinson's diseases, has been associated with alterations in the myelin sheath in the aging brain $(11,12)$. However, the genetic mechanism underlying these age-related alterations in the myelin sheath has not yet been fully elucidated.

Although a number of quantitative studies have investigated the morphological changes that appear in the nervous system with age, they have mainly focused on the alterations in peripheral nerve trunks $(13,14)$, whilst studies on the myelin sheath in the central nervous system (CNS) remain limited.

In the present study the morphological changes in the optic nerve of male Sprague Dawley rats were analyzed at varying time-points between birth and senescence. Age-related profiling of the myelin sheath in the optic nerves of rats was established using microarray hybridization to determine the molecular changes underlying aging in the CNS.

\section{Materials and methods}

Animals. Male Sprague Dawley albino rats (obtained from the Laboratory Animal Center, The Fourth Military Medical University, Xi'an, China) ranging in age from postnatal day (PND) 5 to PND 360 were used. The rats were housed in 
plastic cages with access to food and water ad libitum and maintained on a 12-h light/dark cycle at room temperature $\left(22-26^{\circ} \mathrm{C}\right)$. The experimental protocol was approved by the Institutional Animal Care and Use Committee of The Fourth Military Medical University (Permit no. SCXK2007-007), and the present study was performed in accordance with the National Institutes of Health (NIH) Guide for the Care and Use of Laboratory Animals (NIH Publications No. 80-23).

Electron microscopy analysis. Five rats per group were infused with $2.5 \%$ glutaraldehyde and $4 \%$ paraformaldehyde in $0.1 \mathrm{M}$ phosphate buffer ( $\mathrm{pH}$ 7.4) following anesthetization with sodium pentobarbital $(80 \mathrm{mg} / \mathrm{kg}$; Sigma, St. Louis, MO, USA). The optic nerves were collected and post-fixed using $1 \% \mathrm{OsO}_{4}$ in $0.1 \mathrm{M}$ sodium cacodylate buffer for $2 \mathrm{~h}$ at room temperature and then dehydrated in an ascending acetone series. The osmicated tissue blocks were further embedded in Epon-812 (Serva, Heidelberg, Germany) and trimmed under the light microscope. Ultrathin sections $(50-70 \mathrm{~nm})$ were cut perpendicularly to the axis of nerve fibers using a diamond knife on an LKB-11800 ultramicrotome (LKB, Stockholm, Sweden) and collected by copper grids (300 mesh). The ultrathin sections were stained with uranyl acetate and lead citrate and then observed under an electron microscope (EM; Hitachi, Tokyo, Japan). Microphotograph images were captured at the same time.

Histopathological evaluation. Morphometric evaluation of myelinated fibers (MFs) was performed by measuring $\geq 200$ individual MFs from the sets of photographs selected from five rats at each time-point. The age-related pathological alterations of the myelin sheath were quantified using fiber pathological grading and counting, which were established in our previous study (15). The damaged MFs were classified into three grades according to the severity and extent of destruction, and the percentage of damaged nerve fibers was calculated. The gradings were as follows: I, slight pathological changes, including myelin lamina rarefaction and focal demyelination or vacuolization, with the axon being less affected; II, moderate pathological changes, including myelin lamina reticulation, focal demyelination, vacuolization and axonal changes, such as increased electron density, lipofuscin deposition and glycogen granules; III, severe pathological changes, including marked myelin damage or disruption, accompanied by axonal degeneration and loss.

Microarray hybridization. RNA was extracted from the optic nerves of male normal Sprague Dawley albino rats grouped according to 10 age-points (between PND 5 and PND 360). In order to obtain sufficient RNA for one array hybridization, between three and six samples from one group were pooled as one biological replicate. Independent hybridizations of three biological replicates were performed for each time-point. Following homogenization, total RNA was extracted using TRIzol ${ }^{\circledR}$ reagent (Invitrogen Life Technologies, Carlsbad, CA, USA) in accordance with the manufacturer's instructions. The quantity and quality of total RNA were assessed by measuring the absorbance at 260 and $280 \mathrm{~nm}$ and by gel electrophoresis. Approximately $1 \mu \mathrm{g}$ total RNA was converted to biotin-labeled cRNA and hybridized to the Agilent-014879 whole rat genome microarray 4x44K G4131K (Agilent Technologies, Inc., Santa Clara, CA, USA) in accordance with the manufacturer's instructions.

Quantitative polymerase chain reaction $(q P C R)$. For all tissues, $1 \mu \mathrm{g}$ total RNA was treated with DNase I (Invitrogen Life Technologies) and used to generate cDNA using a BioRT Two Step Reverse Transcription kit (BioER, Hangzhou, China). Resulting cDNA (200 ng) was used as a template for qPCR and RT-PCR in each reaction. The qPCR was performed using the Applied Biosystems 7900HT Fast Real-Time PCR system with the associated Sequence Detection System software version 2.2.2 (Applied Biosystems ${ }^{\mathrm{TM}}$, Foster City, CA, USA). The RT-PCR was performed using a 2x Taq PCR mix kit (Hangzhou Bioer Technology Co., Ltd., Hangzhou, Zhejiang, China). The qPCR and RT-PCR reactions were performed as follows: $95^{\circ} \mathrm{C}$ for $10 \mathrm{sec}, 40$ cycles of $95^{\circ} \mathrm{C}$ for $5 \mathrm{sec}$, and $60^{\circ} \mathrm{C}$ for $34 \mathrm{sec}$. Amplification levels were normalized to expression levels of $\beta$-actin for each sample.

Data analysis. Following normalization, probes that showed significant alterations in expression profiling during the life span were filtered using a one-way analysis of variance (ANOVA) $(\mathrm{P}<0.0001)$. A total of 3,826 genes were selected for further analysis. Hierarchical clustering was performed in those rat transcripts that were expressed differently in the optic nerve among different ages. Each cluster was analyzed based on the gene ontology (GO) function (16). GO annotations were taken from the GO database version 2.2.11. Hypergeometric distributions were used to detect over- or under-represented biological process terms in the studied set compared with the population set. Probabilities obtained using hypergeometric distributions were corrected by Bonferroni correction for the test on multiple GO functions. In order to decrease the number of GO terms, only biological process ontology terms at four, five and six levels were considered. Terms with Bonferroni-corrected $\mathrm{P}$-values $<1 \times 10^{-6}$ were considered to be significant.

\section{Results}

Age-related structural alterations in the myelin sheath in the optic nerve of rats. To determine the effects of aging on the structure of the myelin sheath and the MFs, ultrathin sections prepared from the optic nerve of rats aged between PND 5 and 360 were analyzed under an EM. Marked age-related alterations were observed in the myelin sheaths and MFs of rat optic nerves (Fig. 1A).

In the optic nerve of rats, the process of myelination started after PND 5 and was not fully completed by PND 14. At PND 14 nearly half the fibers in the optic nerve appeared to be unmyelinated axons. The oligodendrocytes of this developing period were activated and contained little heterochromatin, which contributed to the relatively pale appearance of the oligodendrocyte nuclei under the EM. By PND 28, myelination was complete and nearly all the axons were surrounded by a myelin sheath. In this period, the heterochromatin in the nuclei of the oligodendrocytes increased, which is a typical characteristic of ordinary oligodendrocytes. Marked breakdown of myelin occurred in the optic nerve of rats at PND 140, including the myelin tubercles, the general separation of myelin lamellae and 

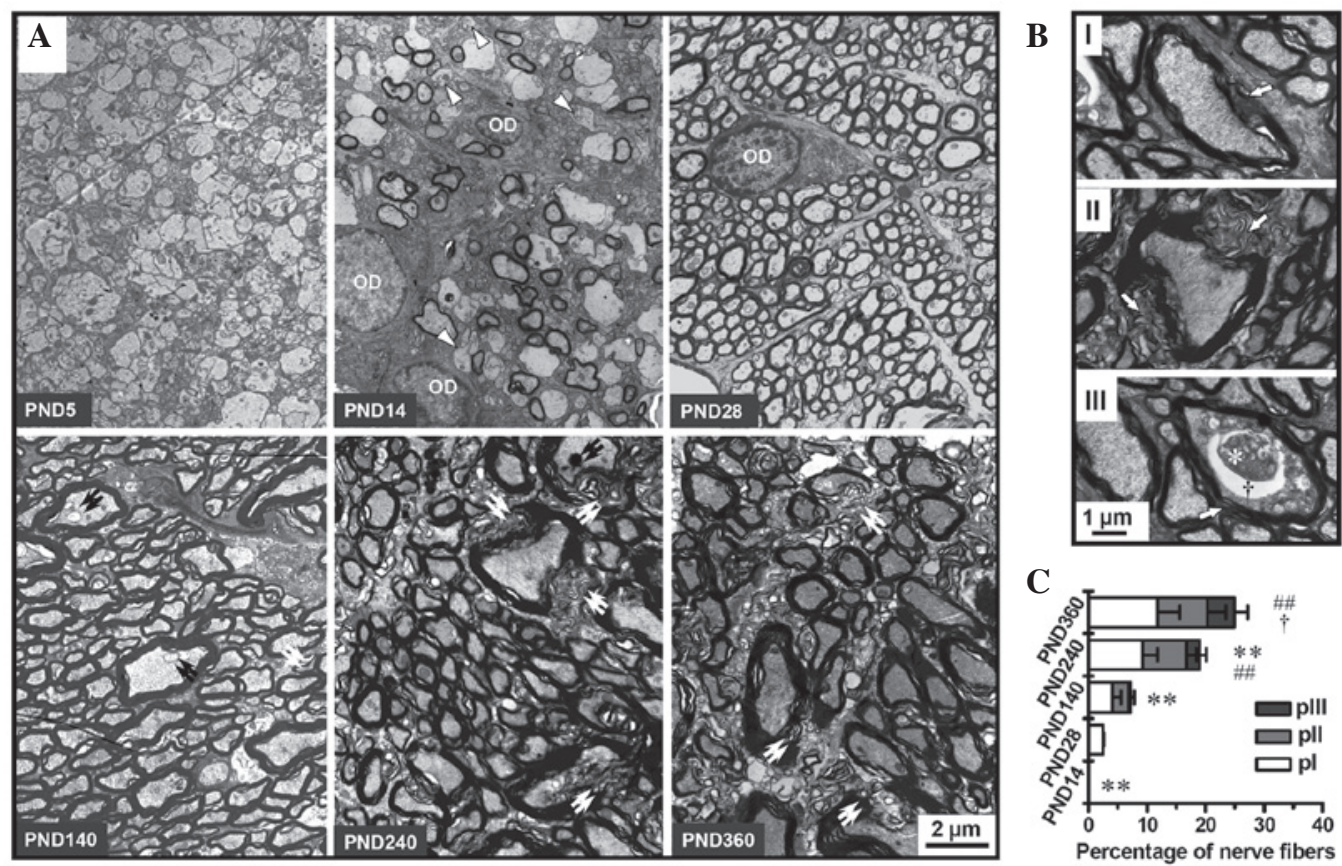

Figure 1. Age-related neuropathies in MFs of the optic nerve of rats. (A) Electron microscopy images of a cross-section (stained with uranyl acetate and lead citrate) of the optic nerve fibers in rats between PND 5 and PND 360 (magnification, x2,500). Nerve fibers are not myelinated at PND 5 and only partial myelination of nerve fibers may be observed at PND 14 in the optic nerve of rats (white triangles). Marked myelin breakdown (white double arrow) and axon degeneration (black double arrow) are present in the aged optic nerve. (B) Grading classification (I-III) of the pathological changes in the optic nerve of rats between PND 14 and PND 360 (magnification, $\mathrm{x} 4,000$ ). (C) Proportions of MFs with different pathologically-classified grades (I-III). ${ }^{* *} \mathrm{P}<0.01$ compared with PND 28; ${ }^{\#} \mathrm{P}<0.01$ compared with PND 140; ${ }^{\circ} \mathrm{P}<0.05$ compared with PND 240. Error bars represent the mean \pm standard error of the mean. MFs, myelinated fibers; PND, postnatal day; OD, oligodendrocyte.

the degeneration of axons. Similar but more extensive deterioration of the myelin was observed in the optic nerves of rats at PND 240 and 360. Severe decompaction of lamellae made the myelin appear wave-like. The matrix between the MFs also underwent a severe decline after PND 240 (Fig. 1A).

Considering the non-statistical alterations in the g-ratio distribution in the optic nerve of rats among different ages (data not shown), grading and counting methods established in our previous study (15) were used to evaluate the age-related changes in the myelin sheath. Grading classification of the pathological changes in the myelin sheath showed the age dependence of the breakdown of the myelin sheath (Fig. 1B and C). The percentage of nerve fibers with pathological alterations in the myelin sheath increased significantly in the optic nerves of aging and aged rats, reaching $25.1 \%$ at PND 360 .

Gene expression data collection and validation. Using the rat whole genome expression microarray, 30 samples of rat optic nerves, from 10 time-points between PND 5 and 360, were analyzed. The coefficients of variation in the data from 30 bio-chips were found to be between 5.9 and $14.8 \%$, whilst the detection rates of each array were between 71.3 and $91.2 \%$. Following normalization, the age-related gene expression profiles were established for the subsequent bioinformatic analyses. To validate the microarray data, the age-related expression levels of certain myelin-associated genes were analyzed using reverse transcription PCR (RT-PCR) and qPCR.

The expression levels of certain transcripts from the microarray data, which were normalized against the PND 5 expression levels, were validated using RT-PCR; the results obtained from these two methods showed concordance (Fig. 2A). Similarly, the expression data from the microarray and the qPCR analysis were also consistent. The slope of the line of best fit between the data from the array and the qPCR was nearly one (Fig. 2B), and the expression of all selected myelin-associated genes showed similar age-related alterations in the microarray and qPCR data (Fig. 2C).

Revealing transcriptional changes underlying optic nerve lifespan. Using high-throughput one-way ANOVA, 3,826 genes that showed differences in expression at each time-point were identified $(\mathrm{P}<0.0001)$. Hierarchical clustering analysis was used on these differentially expressed genes to investigate the categorized characteristics of lifespan and gene expression (Fig. 3). The results suggested that the lifespan of the optic nerve in rats could be divided into three stages: The myelin development period between PND 5 and 14; the maintenances of mature myelin sheath between PND 21 and 56; and the aging process of the myelin sheath, starting from PND 140. Clustering analysis also divided those differentially expressed genes into four subsets according to their expression tendency (Fig. 3). Cluster A included 1,866 transcripts only expressed at high levels during the early development period. Transcripts in cluster B (1,073 transcripts) had low expression levels in development, but had high expression following the mature period. Transcripts in cluster C (204 transcripts) were upregulated during the maintenance period of the mature myelin sheath, but had low expression during the aging period. Cluster D contained 683 transcripts that were identified as aging-specific genes (peak expression emerged only after PND 140). 

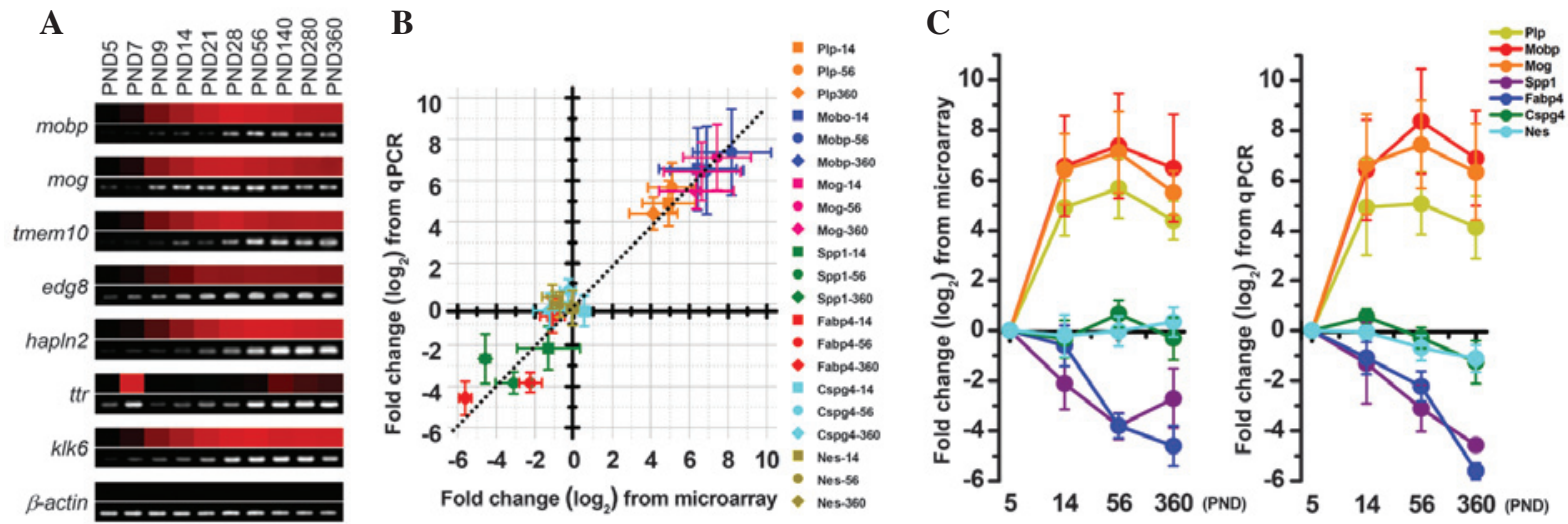

Figure 2. Validation of microarray data using RT-PCR and qPCR. (A) Certain transcripts from the microarray data (colored bars, normalized against PND 5 expression levels) were compared with the RT-PCR results (electrophoresis bands). (B) Correlation between expression data from the microarray and qPCR. The fitting curve is a line $\left(y=0.20+0.93 x\right.$; adjusted $\left.\mathrm{R}^{2}, 0.957 ; \mathrm{P}<0.01\right)$. (C) Transcripts expressing fold change from microarray and qPCR data, respectively. The data from these three methods were consistent. Error bars represent the mean \pm standard error of the mean. RT-PCR, reverse transcription polymerase chain reaction; qPCR, quantitative PCR; PND, postnatal day.

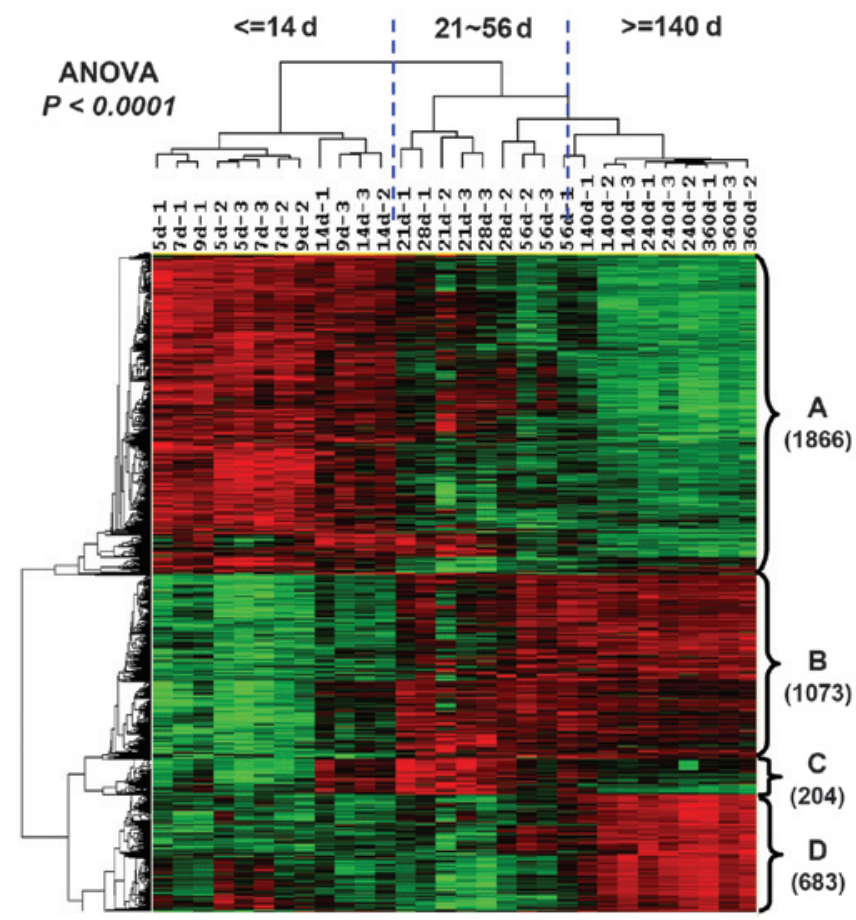

Figure 3. Transcriptional profiling of rat optic nerves of different ages. The hierarchical clustering data of the rat transcripts that were differentially expressed in the optic nerve $(\mathrm{P}<0.0001)$ at different ages are shown. Each row represents the expression level for one gene during development and each column is a time-point. All 3,826 genes are clustered into four clusters (A-D). ANOVA, analysis of variance.

Aging predominantly affects genes involved in lipid biosynthesis, immune response and transmitter transportation. Functional enrichment analysis, along with GO annotation, was used to determine the functions of the genes in each cluster subset. As the results suggest, genes in cluster A, which were highly expressed only during the developmental period, were predominantly involved in proliferation, including the biological processes of cell division, cell cycle, mitosis and DNA replication (cluster A; Fig. 4). The other three clusters, which showed more marked alterations in the aging period, were significantly enriched for genes involved in the immune

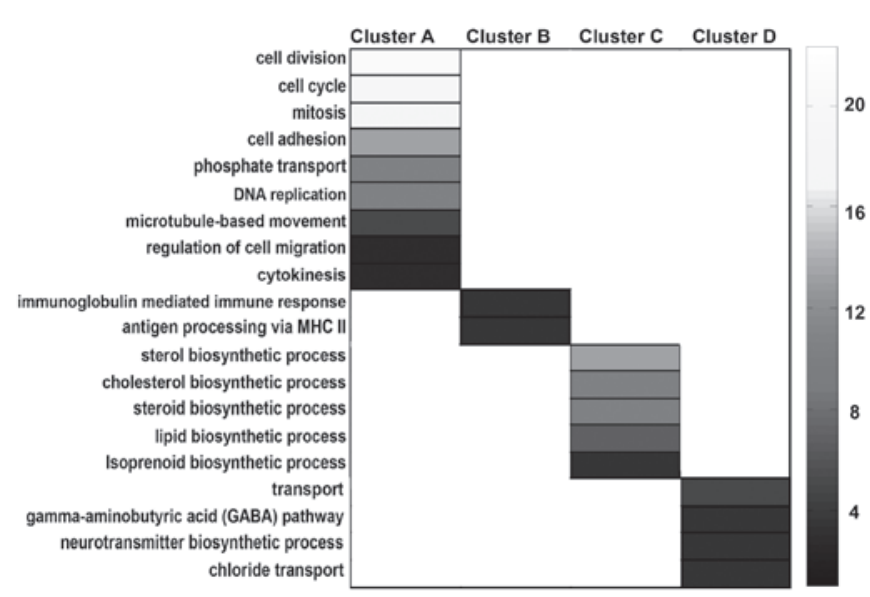

Figure 4. Biological function analysis of genes in clusters A-D. The GO analysis was taken from the GO database version 2.2.11. Enriched functions of genes in each cluster are listed in the figure, with different shades of black/gray indicating the significance level (scaled by the negative log of the corrected P-value). GO, gene ontology.

response, lipid biosynthesis and transmitter transportation, indicating that these three biological processes were the most affected in optic nerve aging (Fig. 4). The induction of immune processes in the mature and aging periods of the myelin sheath included the adaptive immune response and antigen processing and the presentation of exogenous peptide (cluster B; Fig. 4). By contrast, transcripts encoding proteins involved in lipid, sterol and isoprenoid biosynthesis exhibited very low expression in the aging period (cluster C; Fig. 4). The aging-specific genes were found to be transmitter transport genes, particularly those involved in inhibitory transmitter transportations, including the $\gamma$-aminobutyric acid pathway and the chloride transport processes (cluster D; Fig. 4).

\section{Discussion}

To the best of our knowledge, the present study provides the first genome-wide view of changes in gene expression in the optic nerve of aging rats, along with a comparison of myelin sheath structure. The expression database, covering 
10 time-points between development and senescence, is likely to be a valuable resource for further research on the effects of aging on the myelin sheath.

Using the pathological fiber grading and counting method established in our previous study (15), the degree of myelin disruption in the optic nerve of aging rats was quantified, and it was found that the pathological alterations in the myelin sheath were age-dependent. As early as PND 140, mild pathological changes were observed in the myelin sheath in the rat optic nerve fibers. More severe pathological alterations were observed in rats at PND 240, with further deterioration in older rat optic nerves. Similar age-related myelin breakdown has been described in the optic nerve and other regions of the CNS in rhesus monkeys $(10,17,18)$. Although few studies have focused on the effects of aging on the CNS of rats, a number of studies have found similar marked abnormalities of the myelin sheath in the peripheral nervous system (PNS) of aged rats $(14,19)$.

The first genome-wide profiling of age-related changes in peripheral nerves was published in 2012, which indicated an upregulation of immune response transcripts and a downregulation of lipid metabolism transcripts in aging mice (20). By comparing the results from the present study with the previous gene expression profile of the PNS, the genetic mechanisms underlying the age-related myelin sheath decline in the CNS and PNS were able to be identified (20). Although the same GOs were enriched in these two profiles, fewer overlapping genes were found in the two datasets, which suggests differences in aging regulation among different species.

The observed upregulation of transcripts involved in immune response processes following the mature period is in accordance with previous studies, which demonstrated an increase in inflammatory responses during the aging process in the brain $(21,22)$ and the accumulation of macrophages in aging peripheral nerves (13). Our previous data also strongly suggested that there was a correlation between the age-related microglia activation and the age-related myelin breakdown in nearly all parts of the CNS of rats (23). Furthermore, an increase in the number of macrophages has been observed in animal models of inherited neuropathies, and macrophages and/or microglia have been shown to have a pathogenic impact on the myelin sheath through chemokine signaling-mediated demyelination (24). Notably, the activation of the complement cascade has been observed in the brain of aging monkeys $(25,26)$ and the inactivation of the complement cascade has been indicated to facilitate the regeneration of injured nerves (27). These studies further support the relevance of immune response processes as potential targets for drug development for the prevention of age-related myelin breakdown.

The significant over-representation of transcripts involved in lipid synthesis among the downregulated genes in the aging period indicate the importance of this biological process in the vulnerability of the myelin sheath to aging. Lipids constitute $>70 \%$ of myelin membranes and are required in large quantities for myelin assembly (28). The low expression levels of lipid metabolism genes observed after PND 140 are consistent with the detected myelin breakdown in the optic nerve of aging rats (Fig. 1). A similar downregulation of lipid synthesis genes was also identified in the aging profiling of the peripheral nerves of mice and in the profiling of peripheral myelin protein 22-, SREBF chaperone- or Lipin 1-knockout mice (20). The results of the present study, combined with those of previous reports, demonstrate the importance of oligodendrocyte lipids as markers of myelin sheath integrity and function, not only in the period of myelin development, but also during myelin decline. Furthermore, it was found in the present study that the downregulation of lipid metabolism transcripts was paralleled by the reduction in myelin-associated proteins, including myelin basic protein, myelin-related oligodendrocyte basic protein and myelin oligodendrocyte glycoprotein, which have been previously shown to be downregulated in the CNS (23) and PNS of aging rats (29). It is possible that the age-related reduction in cholesterol metabolism genes resulted in the decreased expression of myelin protein genes, as previously observed in a mouse mutant affecting glial cholesterol biosynthesis (30). These observations, together with those of previous reports describing the role of local lipid metabolism in myelin development and function $(30,31)$, strongly indicate that alterations in lipid synthesis transcripts contribute to the age-related pathophysiological changes observed in the myelin sheath.

The upregulation of the transcripts involved in the neurotransmitter transport process, particularly the inhibitory neurotransmitter pathway, was quite novel. Further studies investigating the interrelation between the neurotransmitter transport processes and the age-related myelin decline are required, as, to the best of our knowledge, no previous studies have reported this until now.

In conclusion, in the present study the morphological changes in the myelin sheath in the optic nerve of rats were analyzed at 10 time-points throughout life. Marked alterations in the myelin sheath were observed in the optic nerves of aging and aged rats, which became aggravated with age. Age-related profiling of the myelin sheath in the optic nerves of rats was established using microarray hybridization at 10 time-points throughout life, between birth and senescence. In the present analysis 3,826 transcripts associated with age-induced alterations in the myelin sheath in the optic nerve were identified. It was also found that the most significantly altered biological processes in aging were lipid metabolism, the immune response and transmitter transport. This indicates that the downregulation of lipid synthesis genes and the upregulation of immune genes and neurotransmitter transport genes in aging may provide a genetic basis for the age-related alterations observed in the myelin sheath.

\section{Acknowledgements}

This study was supported by grants from the Major State Basic Research Development Program of China (973 Program) (nos. 2011CB504100 and 2013BAI04B04) and the National Natural Science Foundation of China (no. 81171049).

\section{References}

1. Mold JW, Vesely SK, Keyl BA, Schenk JB and Roberts M: The prevalence, predictors, and consequences of peripheral sensory neuropathy in older patients. J Am Board Fam Pract 17: 309-318, 2004 
2. Mattay VS, Fera F, Tessitore A, et al: Neurophysiological correlates of age-related changes in human motor function. Neurology 58 : 630-635, 2002.

3. Nyberg L, Lövdén M, Riklund K, Lindenberger U and Bäckman L: Memory aging and brain maintenance. Trends Cogn Sci 16: 292-305, 2012.

4. Bartzokis G, Lu PH and Mintz J: Human brain myelination and amyloid beta deposition in Alzheimer's disease. Alzheimers Dement 3: 122-125, 2007.

5. Sherin JE and Bartzokis G: Human brain myelination trajectories across the lifespan: implications for CNS function and dysfunction In: Handbook of the Biology of Aging. Masoro EJ and Austad SN (eds). 7th edition. Academic Press, San Diego, CA, pp333-346, 2011.

6. Kochunov P, Thompson PM, Lancaster JL, et al: Relationship between white matter fractional anisotropy and other indices of cerebral health in normal aging: tract-based spatial statistics study of aging. Neuroimage 35: 478-487, 2007.

7. Verdú E, Ceballos D, Vilches JJ and Navarro X: Influence of aging on peripheral nerve function and regeneration. J Peripher Nerv Syst 5: 191-208, 2000.

8. Peters A: The effects of normal aging on myelinated nerve fibers in monkey central nervous system. Front Neuroanat 3: 11, 2009.

9. Hinman JD and Abraham CR: What's behind the decline? The role of white matter in brain aging. Neurochem Res 32: 2023-2031, 2007.

10. Peters A and Kemper T: A review of the structal alterations in the cerebral hemispheres of the aging rhesus monkey. Neurobiol Aging 33: 2357-2372, 2012.

11. Bartzokis G: Alzheimer's disease as homeostatic responses to age-related myelin breakdown. Neurobiol Aging 32: 1341-1371, 2011.

12. Bohnen NI and Albin RL: White matter lesions in Parkinson disease. Nat Rev Neurol 7: 229-236, 2011.

13. Ceballos D, Cuadras J, Verdú E and Navarro X: Morphometric and ultrastructural changes with ageing in mouse peripheral nerve. J Anat 195: 563-576, 1999.

14. Sharma AK, Bajada S and Thomas PK: Age changes in the tibial and plantar nerves of the rat. J Anat 130: 417-428, 1980.

15. Xie F, Fu H, Hou JF, Jiao K, Costigan M and Chen J: High energy diets-induced metabolic and prediabetic painful polyneuropathy in rats. PLoS One 8: e57427, 2013.

16. Li T, Huang J, Jiang Y, et al: Multi-stage analysis of gene expression and transcription regulation in C57/B6 mouse liver development. Genomics 93: 235-242, 2009.

17. Sandell JH and Peters A: Effects of age on nerve fibers in the rhesus monkey optic nerve. J Comp Neurol 429: 541-553, 2001.
18. Luebke J, Barbas $\mathrm{H}$ and Peters A: Effects of normal aging on prefrontal area 46 in the rhesus monkey. Brain Res Rev 62: 212-232, 2010

19. Majeed SK: Survey on spontaneous peripheral neuropathy in aging rats. Arzneimittelforschung 42: 986-990, 1992.

20. Verdier V, Csárdi G, de Preux-Charles AS, et al: Aging of myelinating glial cells predominantly affects lipid metabolism and immune response pathways. Glia 60: 751-760, 2012.

21. Ginsberg SD: Expression profile analysis of brain aging. In: Brain Aging: Models, Methods, and Mechanisms. Riddle DR (ed). CRC Press, Boca Raton, pp159-185, 2007.

22. Lee CK, Weindruch R and Prolla TA: Gene-expression profile of the ageing brain in mice. Nat Genet 25: 294-297, 2000.

23. Xie F, Zhang JC, Fu H and Chen J: Age-related decline of myelin proteins is highly correlated with activation of astrocytes and microglia in the rat CNS. Int J Mol Med 32: 1021-1028, 2013.

24. Mäurer M, Kobsar I, Berghoff M, Schmid CD, Carenini S and Martini R: Role of immune cells in animal models for inherited neuropathies: facts and visions. J Anat 200: 405-414, 2002.

25. Hinman JD, Duce JA, Siman RA, Hollander W and Abraham CR: Activation of calpain-1 in myelin and microglia in the white matter of the aged rhesus monkey. J Neurochem 89: 430-441, 2004

26. Sloane JA, Hinman JD, Lubonia M, Hollander W and Abraham CR: Age-dependent myelin degeneration and proteolysis of oligodendrocyte proteins is associated with the activation of calpain-1 in the rhesus monkey. J Neurochem 84: 157-168, 2003.

27. Ramaglia V, Tannemaat MR, de Kok M, et al: Complement inhibition accelerates regeneration in a model of peripheral nerve injury. Mol Immunol 47: 302-309, 2009.

28. Chrast R, Saher G, Nave KA and Verheijen MH: Lipid metabolism in myelinating glial cells: lessons from human inherited disorders and mouse models. J Lipid Res 52: 419-434, 2011.

29. Rangaraju S, Hankins D, Madorsky I, et al: Molecular architecture of myelinated peripheral nerves is supported by calorie restriction with aging. Aging Cell 8: 178-191, 2009.

30. Saher G, Quintes S, Möbius W, et al: Cholesterol regulates the endoplasmic reticulum exit of the major membrane protein P0 required for peripheral myelin compaction. J Neurosci 29: 6094-6104, 2009.

31. Verheijen MH, Camargo N, Verdier V, et al: SCAP is required for timely and proper myelin membrane synthesis. Proc Natl Acad Sci USA 106: 21383-21388, 2009. 